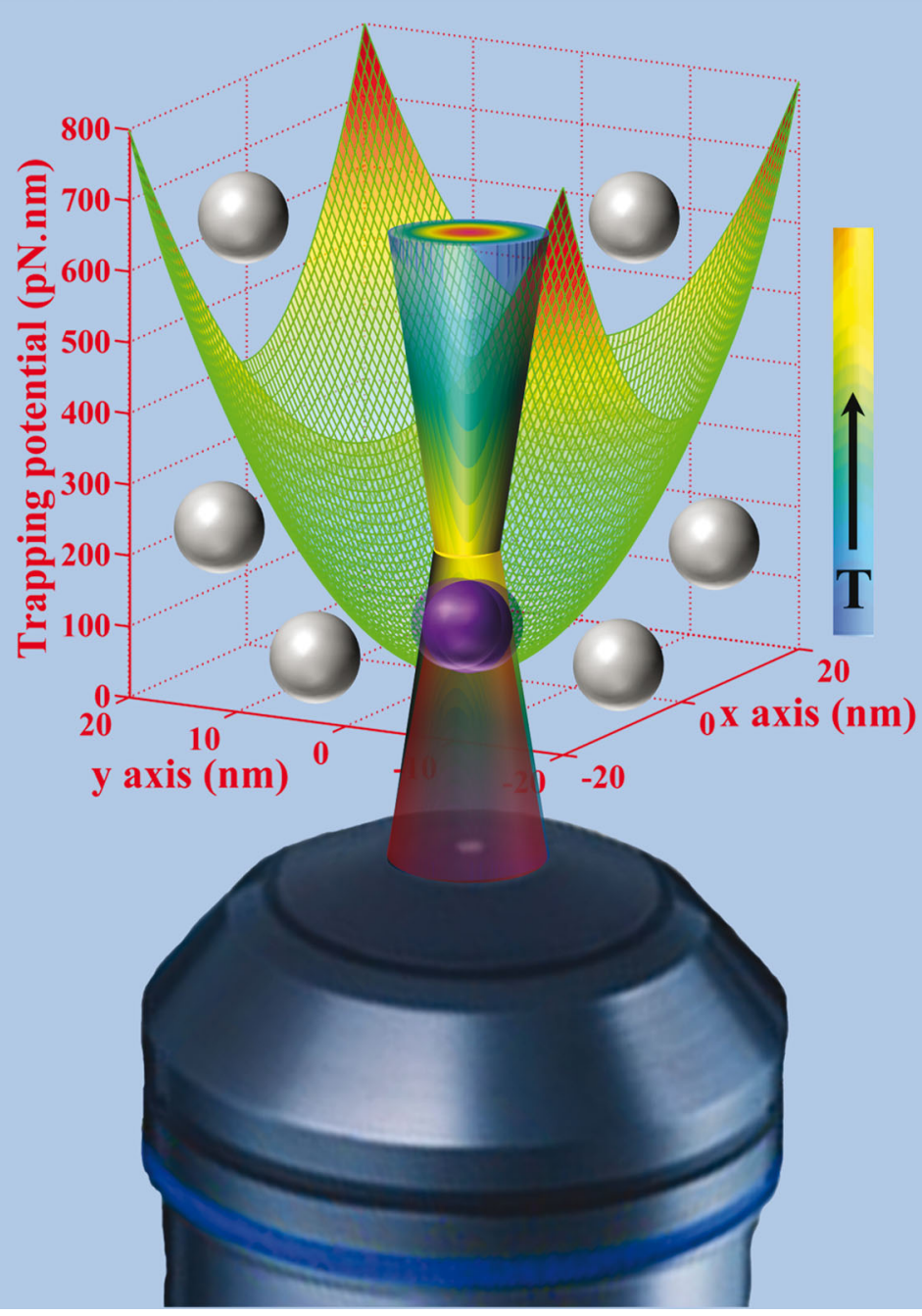

Showcasing research from the Femtosecond Laser Labs in the Department of Chemistry and the Center for Lasers and Photonics at the Indian Institute of Technology Kanpur, India

Title: Precise control and measurement of solid-liquid interfacial temperature and viscosity using dual-beam femtosecond optical tweezers in the condensed phase

This paper reports a novel method of microrheology based on femtosecond optical tweezers, which in turn enables a direct measure and control of in situ temperature at microscale volumes. A polystyrene bead trapped with noninvasive pulsed $780 \mathrm{~nm}$ laser responds spontaneously to changes in its environment induced by a co-propagating $1560 \mathrm{~nm}$ pulsed heating laser. "Hot Brownian motion" of the trapped bead is reflected in the corner frequency as deduced from its power spectrum. Changes in corner frequency values enable calculation of viscosity and temperature at the solid-liquid interface.

\section{As featured in:}

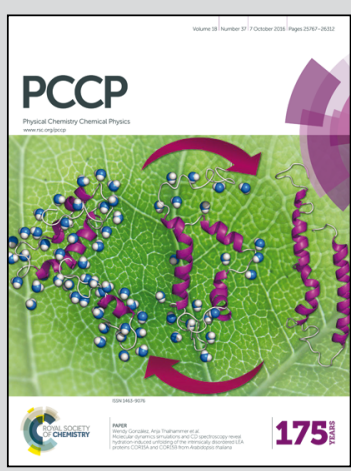

See Debabrata Goswami et al., Phys. Chem. Chem. Phys., 2016, 18, 25823. 


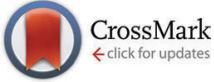

Cite this: Phys. Chem. Chem. Phys., 2016, 18, 25823

\title{
Precise control and measurement of solid-liquid interfacial temperature and viscosity using dual-beam femtosecond optical tweezers in the condensed phase $\uparrow$
}

\author{
Dipankar Mondal, ${ }^{\mathrm{a}}$ Paresh Mathur ${ }^{\mathrm{b}}$ and Debabrata Goswami*ab
}

Received 8th May 2016,

Accepted 29th July 2016

DOI: $10.1039 / \mathrm{c} 6 \mathrm{cp} 03093 a$

www.rsc.org/pccp

\begin{abstract}
We present a novel method of microrheology based on femtosecond optical tweezers, which in turn enables us to directly measure and control in situ temperature at microscale volumes at the solid-liquid interface. A noninvasive pulsed $780 \mathrm{~nm}$ trapped bead spontaneously responds to changes in its environment induced by a co-propagating $1560 \mathrm{~nm}$ pulsed laser due to mutual energy transfer between the solvent molecules and the trapped bead. Strong absorption of the hydroxyl group by the $1560 \mathrm{~nm}$ laser creates local heating in individual and binary mixtures of water and alcohols. "Hot Brownian motion" of the trapped polystyrene bead is reflected in the corner frequency deduced from the power spectrum. Changes in corner frequency values enable us to calculate the viscosity as well as temperature at the solid-liquid interface. We show that these experimental results can also be theoretically ratified.
\end{abstract}

\section{Introduction}

Optical tweezers ${ }^{1,2}$ are being used in various fields of research for numerous applications. The principle of optical trapping is based on radiation pressure exerted by light and is mainly used to hold and manipulate various objects with sizes ranging from several nanometers to a few microns. We use $780 \mathrm{~nm}$ femtosecond pulsed optical tweezers ${ }^{3-5}$ that provide a high gradient force from only $7 \mathrm{~mW}$ average laser power. Such a high peak-power Gaussian laser beam can easily trap and manipulate small microparticles, nanoparticles or even quantum dots. ${ }^{6}$ This trapping wavelength is transparent through water, alcohol, most of the bio-molecule solutions, cells, and is confirmed to be safe and non-invasive due to its very low absorption coefficient. ${ }^{7}$ Consequently, the temperature rise is negligible for the low laser powers used in such experiments. On the other hand, there is significant evidence of temperature rise for a $1560 \mathrm{~nm}$ pulsed laser because of the strong absorption coefficient at the $\nu_{1}+\nu_{3}$ combination band (i.e., two fundamental vibrational bands are simultaneously excited). ${ }^{8-10}$ The vibrational combination band near $1560 \mathrm{~nm}$ of hydroxyl, amine and thiol can also be useful for investigating viscosity changes from the temperature rise. Earlier, stable $3 \mathrm{D}$ trapping of cells, viruses, bacteria, etc. has

\footnotetext{
${ }^{a}$ Indian Institute of Technology Kanpur, Department of Chemistry, Kanpur 208016, Uttar Pradesh, India. E-mail: dgoswami@iitk.ac.in

${ }^{b}$ Indian Institute of Technology Kanpur, Center for Lasers and Photonics, Kanpur 208016, Uttar Pradesh, India

$\dagger$ Electronic supplementary information (ESI) available. See DOI: 10.1039/c6cp03093a
}

been demonstrated at a $1064 \mathrm{~nm}$ continuous wave $(\mathrm{cw})$ laser with low absorption under experimental conditions. ${ }^{11-14}$ However, the effects on biological systems due to high power $1064 \mathrm{~nm}$ absorption arising from the vibrational combination band $\left(2 v_{2}+v_{3}\right)^{15,16}$ of water have also remained a subject of interest. Thus, the possibility of using a single beam laser trap to simultaneously measure thermal effects has its shortcomings.

We have instead devised a two-colour approach, which in contrast uses one laser at $1560 \mathrm{~nm}$ as an independent source of temperature control that is being simultaneously detected by another co-propagating stable laser trap at $780 \mathrm{~nm}$. The co-propagated non-invasive trapping laser at $780 \mathrm{~nm}$ probes the effect of the pulsed $1560 \mathrm{~nm}$ laser. The main uniqueness of this work over already existing methods ${ }^{17-19}$ lies in the use of a femtosecond optical trap and a heating laser rather than cw lasers, as well as in the inclusion of a correction term for the temperature change. The effects of surroundings on biosystems have often impacted the efficiencies of their physical activities. In this regard, viscosity $(\eta)$ and temperature $(T)$ are two such physical parameters that have a strong control on the nature of bio-molecular activity. ${ }^{20-25}$

We probe the Brownian motion ${ }^{26,27}$ of a spherical polystyrene bead suspended in condensed media. Due to Brownian motion, there exists a characteristic frequency of the trapped particle for every trapping laser power, beyond which the particle cannot remain trapped. This characteristic frequency is known as the corner frequency. Our main focus from the newly developed technique is to measure the corner frequency at different heating laser powers, wherein the spherical polystyrene bead 
spontaneously equilibrates itself to the surrounding temperature. The nature of Brownian motion can be understood by the theory developed by Einstein. ${ }^{28}$ The energy transfer occurs between the trapped bead and the solvents resulting in the transformation of thermal energy of solvents into the kinetic energy of the trap bead. Thus the hot solvent molecules resulting from $1560 \mathrm{~nm}$ absorption can continuously dissipate heat to the trapped stationary bead which will be reflected in the frequency spectrum of Brownian noise exhibited by the bead. The stochastic motion ${ }^{29}$ of a spherical bead confined in harmonic trapping potential under a Gaussian laser pulse having thermal molecular motion will give rise to diffusion, which increases with temperature. Interestingly, the simultaneous increase of the diffusion coefficient and corner frequency will keep the net trap stiffness constant. However, the distinct nature of individual trapped particles may provide an independent measure that would elucidate the absolute instantaneous temperature rise at the trapped bead surface-solvent layer. Temperature dependence of viscosity enables us to phenomenologically solve the expression of nonlinear equation for binary liquid mixtures using numerical methods. Our microrheological $^{30-33}$ investigations on the temperature and viscosity of individual and binary solvent media of water and alcohols can be used to map the environmental temperature or viscosity ${ }^{34}$ at the solid-liquid interface around the confined space of the micro to nano size regime.

This contactless temperature measurement in the sub-microscale volume also leads to the inception of a new micro-viscometer, which can be used to probe the physical properties of confined systems and the effect of surrounding on their behaviour. ${ }^{35}$ Furthermore, we can apply our method to elucidate the structural responses of trapped protein macromolecules ${ }^{36}$ in different solvents and at different temperatures.

Here we demonstrate the measure of local temperature as well as viscosity in water-methanol and water-ethanol binary mixtures both theoretically and experimentally. Our initial model was based only on conduction; ${ }^{9,37}$ thereafter, we observed a deviation between the calculated and experimental data towards higher temperatures. ${ }^{38,39}$ We show here that it is possible to match the experimental results better with a modified model for such liquid mixtures, when we take advantage of our pure sample data. The observed deviation in water has helped us to introduce a new correction term in the theoretical model. With this approach, our method can be extended to any complex fluid media of solvent mixtures having $\mathrm{N}-\mathrm{H}, \mathrm{S}-\mathrm{H}, \mathrm{O}=\mathrm{C}-\mathrm{O}-\mathrm{H}$, and $\mathrm{P}-\mathrm{H}$ groups.

\section{Methods and materials}

In our optical tweezers setup (Fig. 1), the laser source used is an Er-doped fibre laser (Femtolite C-20-SP, IMRA Inc., USA), which generates femtosecond laser pulses centred at fundamental $1560 \mathrm{~nm}$ wavelength and at its second harmonic $780 \mathrm{~nm}$ with pulse-widths $300 \mathrm{fs}$ and $100 \mathrm{fs}$, respectively. The two laser pulse outputs are collinear at a repetition rate of $50 \mathrm{MHz}$. A commercial oil immersion objective (UPlanSApo, $100 \times, 1.4$ NA, OLYMPUS Inc., Japan) was

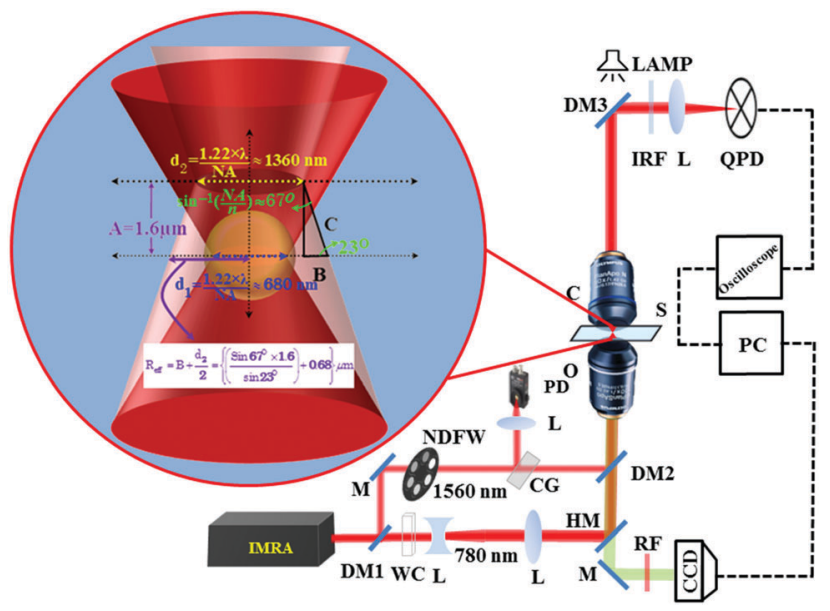

Fig. 1 Optical setup with labels: DM (1-3): dichroic mirror; M: mirror; CG: cover glass; NDFW: neutral density filter wheels; L: lens; PD: photodiode; WC: water cuvette; HM: hot mirror; O: objective lens; S: sample chamber; C: condenser lens; IRF: infrared filter; RF: red filter; QPD: quadrant photodiode; CCD: camera (charge coupled device). Ray optics diagram (inset) (see S1, ESI†).

used to achieve tight focusing; simultaneously the forward scattered light was collected with another oil immersion objective (60×, PlanApo N, 1.42 NA, OLYMPUS Inc., Japan) and focused onto a quadrant photodiode (QPD) (2901, Newport Co., USA). The QPD output was connected to a digital oscilloscope (Waverunner 64Xi, LeCroy, USA) interfaced with a personal computer through a GPIB card (National Instruments, USA). Data were acquired using the LabVIEW program. Spectroscopic grade methanol $(\mathrm{MeOH})$ and ethanol $(\mathrm{EtOH})$ were purchased from Merck, India, and were used without any further purification. We used a $500 \mathrm{~nm}$ mean radius (T8883, Life Technology, USA) fluorophore coated polystyrene sphere suspended in $\mathrm{H}_{2} \mathrm{O}-\mathrm{MeOH}$ and $\mathrm{H}_{2} \mathrm{O}-\mathrm{EtOH}$ mixtures. The commercially available polystyrene nanosphere solution (T8883: concentration $3.6 \times 10^{10}$ particles per $\mathrm{ml}$ ) was diluted to nano-molar concentration and well sonicated for immediate use in trapping experiments. The video of the trapping events was monitored using a CCD camera (350 K pixel, e-Marks Inc., USA). White light was used for bright field illumination. Trapping laser power was measured using a power meter (FieldMate, Coherent, USA) as well as a silicon amplified photodiode (PDA100A-EC, Thorlabs, USA) and $1560 \mathrm{~nm}$ power was measured using a calibrated biased InGaAs detector (DET10C/M, Thorlabs, USA). All laser power measurements with the power-meter were made just before the sample chamber. The absorption spectrum was collected using an absorption spectrometer (Lambda 900, PerkinElmer, USA). A linear motorized stage (UE1724SR driven by ESP300, Newport Co., USA) was used, which was interfaced with a personal computer through the GPIB card. We used a mechanical shutter (SR475), controlled through LABVIEW programming, operating at the maximum rate of $100 \mathrm{kHz}$ to result in an opening time that is shorter than or comparable to the thermal relaxation time. The thermal relaxation time is defined as the average time required to achieve maximum 
temperature at the surface of the bead. ${ }^{40}$ The entire experiment has been done twice to ensure that at least 10 data points are averaged for each time.

\section{Results and discussion}

\subsection{Theoretical section}

When two different colour light beams are focused by the same objective they do not focus at the same plane. Our proposed model (see S1, ESI $\dagger$ ) is able to calculate the effective fluence of the $1560 \mathrm{~nm}$ pulse laser at the focus of the $780 \mathrm{~nm}$ laser, where the polystyrene particle is trapped. This new approach overcomes the difficulty of two different colour pulsed laser fluence calculation of any desired plane though they focus at different positions with $1.6 \mu \mathrm{m}$ separation when focused by the same objective as shown in the Experimental section (Fig. 1). We measured this distance between two colour focal points by placing a thin cover slip sample chamber containing $10^{-4} \mathrm{M}$ Rhodamine-6G in water. The maximum two photon fluorescence (TPF) is emitted from the focal plane of the $780 \mathrm{~nm}$ laser. This epifluorescence is measured on a CCD at the back focal plane (Fig. 1). When the illuminated sample is moved with respect to the $780 \mathrm{~nm}$ focal plane with a linear motorized stage (of minimum resolution $0.00001 \mathrm{~mm}$ ), the minimum transmittance signal occurs on the InGaAs $1560 \mathrm{~nm}$ detector at a position $1.6 \mu \mathrm{m}$ further, which is the focal plane of $1560 \mathrm{~nm}$. For this calibration measurement, we placed an aperture just before the InGaAs detector, which replaces DM3 in Fig. 1. Using the "Law of sines", ${ }^{41}$ the fluence of the $1560 \mathrm{~nm}$ pulsed laser at the focus of $780 \mathrm{~nm}$ trapping laser can be calculated by the following equation (see S1, ESI†):

$$
F_{0}=\frac{\text { Pulse energy }}{\pi\left[\left\{\left(\frac{\sin 67^{\circ}}{\sin 23^{\circ}} \times 1.6\right)+0.68\right\} \times 10^{-6}\right]^{2}}
$$

The half angle of the objective does not change since the focal length of the $100 \times$ objective is higher (above 100 times) than the separation between the two focal points of two colours (Fig. 1). We have thus effectively used the geometric model as a reasonable approximation. The half angle of the cone formed by the focusing laser beam is $\sim 67^{\circ}$ calculated from the relation $\mathrm{NA}=n \sin \alpha$, using 1.4 numerical aperture (NA) of the objective lens and a refractive index $(n)$ of 1.52 for the immersion oil. Using the formula ${ }^{42}$ focal spot $=(1.22 \times \lambda) / \mathrm{NA}$, where $\lambda$ is the laser wavelength, we calculate the beam waist of $780 \mathrm{~nm}$ and $1560 \mathrm{~nm}$ lasers at focus as $680 \mathrm{~nm}$ and $1360 \mathrm{~nm}$ respectively. The increase in temperature with respect to room temperature $\left(T_{0}\right)$ due to the absorption of a $1560 \mathrm{~nm}$ Gaussian beam by solvent media can be evaluated using the following equation: ${ }^{43}$

$$
T_{\max }-T_{0}=0.783 \frac{F}{\tau_{\mathrm{p}}} \frac{2 \sqrt{\tau_{\mathrm{p}}}}{\sqrt{\pi K \rho C}}
$$

The total fluence $(F)$ absorbed by solvents used is calculated from $F=A \times\left(1-\left|r_{\mathrm{f}}\right|^{2}\right) F_{0}$, where $F_{0}$ is the laser fluence before the sample chamber and $r_{\mathrm{f}}$ is the reflection coefficient, which is

\begin{tabular}{|c|c|c|c|c|}
\hline Sample & $\begin{array}{l}\text { Density }(\rho) \\
\left(\mathrm{kg} \mathrm{m}^{-3}\right)\end{array}$ & $\begin{array}{l}\text { Heat } \\
\text { capacity }(C) \\
\left(\mathrm{J} \mathrm{kg}^{-1} \mathrm{~K}^{-1}\right)\end{array}$ & $\begin{array}{l}\text { Thermal } \\
\text { conductivity }(\kappa) \\
\left(\mathrm{W} \mathrm{K}^{-1} \mathrm{~m}^{-1}\right)\end{array}$ & $\begin{array}{l}\text { Absorbance } \\
(A)\end{array}$ \\
\hline $\mathrm{H}_{2} \mathrm{O}$ & $997^{a}$ & $4180^{c}$ & $0.600^{e}$ & \\
\hline $\mathrm{MeOH}$ & $786^{b}$ & $2250^{d}$ & $0.206^{e}$ & \\
\hline EtOH & $785^{b}$ & $2440^{c}$ & $0.178^{e}$ & \\
\hline $\begin{array}{l}\mathrm{H}_{2} \mathrm{O}-\mathrm{EtOH} \\
\text { mixture }\end{array}$ & 990 & 4120 & 0.586 & 0.113 \\
\hline $\begin{array}{l}\mathrm{H}_{2} \mathrm{O}-\mathrm{MeOH} \\
\text { mixture }\end{array}$ & 987 & 4090 & 0.581 & 0.117 \\
\hline
\end{tabular}

Table 1 The used physical parameters of the trapping solvent

calculated from the cover glass refractive index, $n$, using the relation $r_{f}=\left|\frac{n-1}{n+1}\right|$. " $A$ ", is the measured absorbance of binary mixtures in the thin sample chamber. The other parameters used in eqn (2) (see S2, ESI $\dagger$ ) are density ( $\rho$ ), thermal conductivity $(\kappa)$, and heat capacity $(C)$ for binary mixtures that can be calculated from the following set of equations, using pure component physical parameters (Table 1$)^{: 44-48}$

$$
\left.\begin{array}{l}
\rho_{\text {mix }}=\sum_{i=1}^{j} X_{i} \rho_{i} \\
\kappa_{\text {mix }}=\sum_{i=1}^{j} X_{i} \kappa_{i} \\
C_{\text {mix }}=\sum_{i=1}^{j} X_{i} C_{i}
\end{array}\right\}
$$

In eqn (3) the index $j$ is 2 for binary mixtures, whereas the index $i$ represents the pure component of binary mixtures. The mole fraction $(X)$ is calculated from the volume fraction $(\varphi)$ and molecular weight $(M)$ and density $(\rho)$. We note that use of such an ideal behaviour is impractical for viscosity, which is dependent on intermolecular interactions. The viscosity of binary mixtures is calculated based on the temperature rise (Tables 2 and 3) using the following equations: ${ }^{49-51}$

$$
\begin{gathered}
\ln \eta_{\text {binary mix }}=\varphi_{1} \ln \eta_{1}+\varphi_{2} \ln \eta_{2}+\Delta \ln \eta^{\text {excess }} \\
\eta_{\text {binary mix }}=\exp \left\{\left(0.9 \times \ln \left(2.414 \times 10^{-2} \times 10^{\frac{247.8}{140-T}}\right)\right)\right. \\
\left.+\left(0.1 \times\left(A_{i}+\frac{B_{i}}{C_{i}+T}\right)\right)+\Delta \ln \eta^{\text {excess }}\right\}
\end{gathered}
$$

Here $\Delta \ln \left(\eta^{\text {excess }}\right)$ is the excess viscosity due to mixing, which is calculated by using the method developed by Wolf et al. ${ }^{52,53}$ For each individual alcohol, $A, B$ and $C$ are Vogel's equation parameters (see S3, ESI $\dagger$ ).

\subsection{Experimental section}

We have used a pulsed laser at $780 \mathrm{~nm}$ with $7 \mathrm{~mW}$ of average power to trap the polystyrene bead of $500 \mathrm{~nm}$ mean radius. The solvent media used are $\mathrm{H}_{2} \mathrm{O}-\mathrm{EtOH}$ and $\mathrm{H}_{2} \mathrm{O}-\mathrm{MeOH}$ mixtures. In both the cases, a volume ratio of $90: 10$ is used. The trapping laser can be considered non-invasive due to very low absorption 
Table 2 Theoretical and experimental data for a 90:10 (volume-wise) $\mathrm{H}_{2} \mathrm{O}$-EtOH mixture

\begin{tabular}{|c|c|c|c|c|c|c|c|c|}
\hline \multirow[b]{2}{*}{$\begin{array}{l}1560 \mathrm{~nm} \\
\text { Power }(\mu \mathrm{W})\end{array}$} & \multirow[b]{2}{*}{$\begin{array}{l}\text { Corner } \\
\text { frequency }(\mathrm{Hz})\end{array}$} & \multirow[b]{2}{*}{$\begin{array}{l}\text { Fluence } \\
\left(\mathrm{J} \mathrm{m}^{-2}\right)\end{array}$} & \multicolumn{4}{|c|}{ Theoretical data } & \multicolumn{2}{|c|}{ Experimental data } \\
\hline & & & $\begin{array}{l}T_{\max }-T_{0} \\
(\mathrm{~K})\end{array}$ & $\begin{array}{l}\text { Viscosity } \\
(\mathrm{Pa} \mathrm{s}) \times 10^{-3}\end{array}$ & $\begin{array}{l}\text { Corrected } \\
\Delta T_{\text {theo }}\end{array}$ & $\begin{array}{l}\text { Corrected viscosity } \\
(\mathrm{Pa} \mathrm{s}) \times 10^{-3}\end{array}$ & $\begin{array}{l}\text { Temperature } \\
\text { rise }(\mathrm{K})\end{array}$ & $\begin{array}{l}\text { Viscosity } \\
(\mathrm{Pa} \mathrm{s}) \times 10^{-3}\end{array}$ \\
\hline 0 & 83 & 0 & 0 & 1.60 & 0 & 1.60 & 0 & 1.60 \\
\hline 209 & 88 & 0.0672 & 7.6 & 1.32 & 5.5 & 1.35 & 2.2 & 1.51 \\
\hline 363 & 96 & 0.1167 & 13.2 & 1.23 & 9.6 & 1.29 & 6.1 & 1.38 \\
\hline 420 & 110 & 0.1351 & 15.3 & 1.19 & 11.1 & 1.25 & 12.5 & 1.20 \\
\hline 630 & 119 & 0.2026 & 22.9 & 1.10 & 16.6 & 1.18 & 16.2 & 1.11 \\
\hline 830 & 133 & 0.2669 & 30.2 & 1.02 & 21.9 & 1.10 & 21.5 & 0.99 \\
\hline
\end{tabular}

Table 3 Theoretical and experimental data for a $90: 10$ (volume-wise) $\mathrm{H}_{2} \mathrm{O}-\mathrm{MeOH}$ mixture

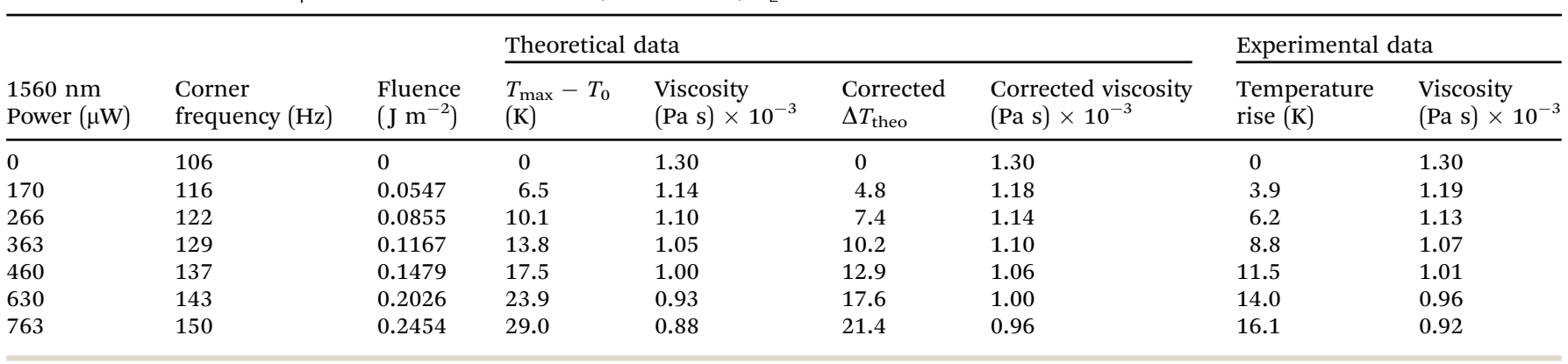

coefficients of the solvents used. ${ }^{7}$ Simultaneously, we also irradiated our trapped volume by a $1560 \mathrm{~nm}$ IR laser where binary solvents have high absorption coefficients resulting in local heating around the trapped particle. The beam waist spot size of the trapping laser is $680 \mathrm{~nm}$ and that of the heating laser is $1360 \mathrm{~nm}$ whereas the effective heating beam radius at the trapping plane is out of the Rayleigh range ${ }^{54}\left(\pi \omega_{0}{ }^{2} / \lambda=931 \mathrm{~nm}\right)$ as the separation is $1.6 \mu \mathrm{m}$ (see Theoretical section for details). Due to the temperature rise, the trapped $500 \mathrm{~nm}$ mean radius particle will show a different behaviour, which is reflected in its "Hot Brownian motion". ${ }^{55-57}$ We measure the temperature change between the polystyrene bead surface and the surrounding liquid layer (through the corner frequency shift) to indicate that thermal flow exists, and the bead displacement due to this flow is coupled with that of a regular confined Brownian motion. We only correlate the measured displacement of the confined Brownian motion as long as our temperature change is not very large according to our model. We have thus probed the confined Brownian motion of the polystyrene bead trapped in a harmonic potential ${ }^{58}$ generated by a Gaussian laser pulse, which can be described by the Langevin equation ${ }^{59}$

$$
m \ddot{X}(t)+\gamma \dot{x}(t)+\kappa_{T S} x(t)=\zeta_{\text {therm }}(t)
$$

where $m$ denotes the particle mass, $x$ signifies the time dependent position, $\gamma$ is the viscous drag coefficient as per Stokes' law, $\kappa_{\mathrm{TS}}$ is the spring constant (or trap stiffness) and $\zeta_{\text {therm }}$ is the time dependent random force. By solving the above equation, we can fit our experimental one-sided power spectrum. Typically, in the power spectrum, power is measured in the interval $f$ and $f+\mathrm{d} f$, which does not distinguish between $+f$ and $-f$. In such cases, it is possible to define one-sided power spectral density ${ }^{60}$ (PSD) (see S4, ESI $\dagger$ ) by the following Lorentzian: ${ }^{61}$

$$
p_{x}(f)=\left\langle p_{x}(f)^{(\exp )}\right\rangle=\frac{A}{\left(f_{\mathrm{c}}^{2}+f^{2}\right)}=\frac{D}{\pi^{2}\left(f_{\mathrm{c}}^{2}+f^{2}\right)}
$$

Here $A$ is a fitting parameter which has information about $D$, the diffusion coefficient. " $A$ " has the unit $V^{2} \mathrm{~s}^{-1}$. The diffusion coefficient can be evaluated from $A$ using the voltage to position calibration $^{62,63}$ factor. We have analysed the forward scattered data of the trapped bead that have been collected with a QPD at a sampling rate of $20 \mathrm{kHz}$ for the first 2.5 seconds of trapping. This is to minimize the convection effects. The acquired data of channels $X$ and $Y$ are de-correlated by removing the crosstalk $^{61,64}$ between them. The processed data are then fitted with eqn (7) to obtain the respective corner frequency within different solvents and at different $1560 \mathrm{~nm}$ laser heating powers.

We observed an increasing trend in the corner frequency $\left(f_{\mathrm{C}}\right)$, which is deduced from the power spectrum (Fig. 2a and b), with an increase in $1560 \mathrm{~nm}$ laser power. From our theoretical model (see eqn (2)), we notice that there is a linear relationship between $1560 \mathrm{~nm}$ laser power and the corresponding temperature rise. Simultaneously, from eqn (4) and (5), we note that viscosity decreases with an increase in $1560 \mathrm{~nm}$ power. Within our experimental range, when the viscosity change with respect to the temperature change lies in the linear regime, we have considered the trap stiffness $\left(\kappa=2 \pi f_{\mathrm{C}} \gamma\right)$ as a constant parameter. This statement is correct as long as there is a continuous change in viscosity with the heating laser source for a constantsized trapped particle in the same solvent medium (see S5, ESI $\dagger$ ). This has enabled us to measure (Tables 2 and 3) the viscosity change and the temperature rise around the interface of the trapped bead and the surrounding solvent molecules from 

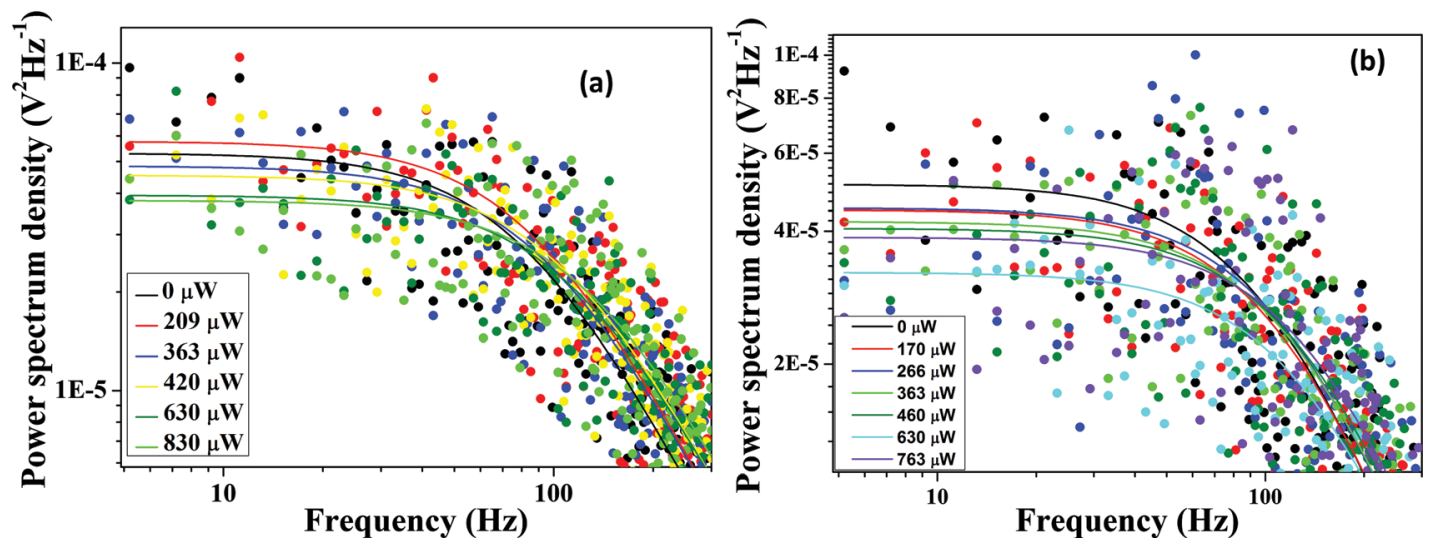

Fig. 2 Experimentally measured power spectrum (scatter points) and the respective Lorentzian fitted data (solid line) for a $500 \mathrm{~nm}$ radius fluorophore coated polystyrene bead in the (a) water-ethanol mixture and (b) water-methanol mixture.

the formula $\eta \cdot f_{\mathrm{C}}=$ constant. $^{19,65}$ All our experimental measurements are performed with respect to the room temperature viscosity at the corresponding corner frequency in individual binary solvents when the $1560 \mathrm{~nm}$ laser is absent.

Experimentally, we first observe the corner frequency change, which allows us to calculate the viscosity around the trapped bead. From our experimentally measured viscosity, we calculate the temperature rise of the respective binary mixtures (Tables 2 and 3) by rearranging the variables in eqn (5) and solving with MATLAB ${ }^{\mathbb{R}}$. The particle experiencing viscous drag, $\gamma=6 \pi \eta r(r=500 \mathrm{~nm})$, has trap stiffness, $\kappa_{\mathrm{TS}}$, for $\mathrm{H}_{2} \mathrm{O}-\mathrm{EtOH}$ and $\mathrm{H}_{2} \mathrm{O}-\mathrm{MeOH}$ binary mixtures as $0.0078 \pm 0.0002 \mathrm{pN} \mathrm{nm}^{-1}$ and $0.0081 \pm 0.0002 \mathrm{pN} \mathrm{nm}^{-1}$ respectively. The confidence range of our trap stiffness (see S5, ESI $\dagger$ ) results is obtained by the standard deviation of all stiffness measurements at different temperatures. As expected, viscosity changes around the trapped bead with respect to temperature changes are linear (Fig. 3a). Our experimental data in Tables 2 and 3 show that the temperature rise between the theoretical calculation and the experimental measurement deviates with the increase in temperature, which is also true for viscosity. This is due to the fact that besides conduction, convection also plays an important role in heat transfer towards high temperature, since with temperature rise, the focal plane separation changes. The refractive index changes as a result of the diverging optical lens ${ }^{66}$ formed in solvent media with the increase in temperature at the focal volume. Additionally, due to the high NA objective, there could be a critical angle effect, which could consistently result in an overestimation of our theoretically estimated temperature change. Consequently, the separation of focal points for the two laser wavelengths becomes slightly more, which reduces the temperature at the focal volume around the optical trap and this is reflected in our experimental results. To account for this, we add a correction term to eqn (2) from our earlier study (see S6, ESI $\dagger$ ) and generalize it for use in binary mixtures:

$$
\left.\begin{array}{rl}
T_{\max }-T_{0}= & 0.783 \frac{F}{\tau_{\mathrm{p}}} \frac{2 \sqrt{\tau_{\mathrm{p}}}}{\sqrt{\pi K \rho C}}-\left\{x_{1}\left[\left(\frac{\mathrm{d} T}{\mathrm{~d} P}\right)_{\text {Theo }}-\left(\frac{\mathrm{d} T}{\mathrm{~d} P}\right)_{\operatorname{Exp}}\right]_{\mathrm{C} 1}\right\} \\
& \left.+\left(1-x_{1}\right)\left[\left(\frac{\mathrm{d} T}{\mathrm{~d} P}\right)_{\text {Theo }}-\left(\frac{\mathrm{d} T}{\mathrm{~d} P}\right)_{\operatorname{Exp}}\right]_{\mathrm{C} 2}\right\} \times P_{\mathrm{avg}}
\end{array}\right\}
$$

Since water is the dominant species in our particular experiments, we can simplify eqn (8) further as follows:

$$
T_{\max }-T_{0}=0.783 \frac{F}{\tau_{\mathrm{p}}} \frac{2 \sqrt{\tau_{\mathrm{p}}}}{\sqrt{\pi K \rho C}}-\left[\left(\frac{\mathrm{d} T}{\mathrm{~d} P}\right)_{\text {Theo }}-\left(\frac{\mathrm{d} T}{\mathrm{~d} P}\right)_{\mathrm{Exp}}\right]_{\mathrm{C} 1} \times P_{\mathrm{avg}}
$$
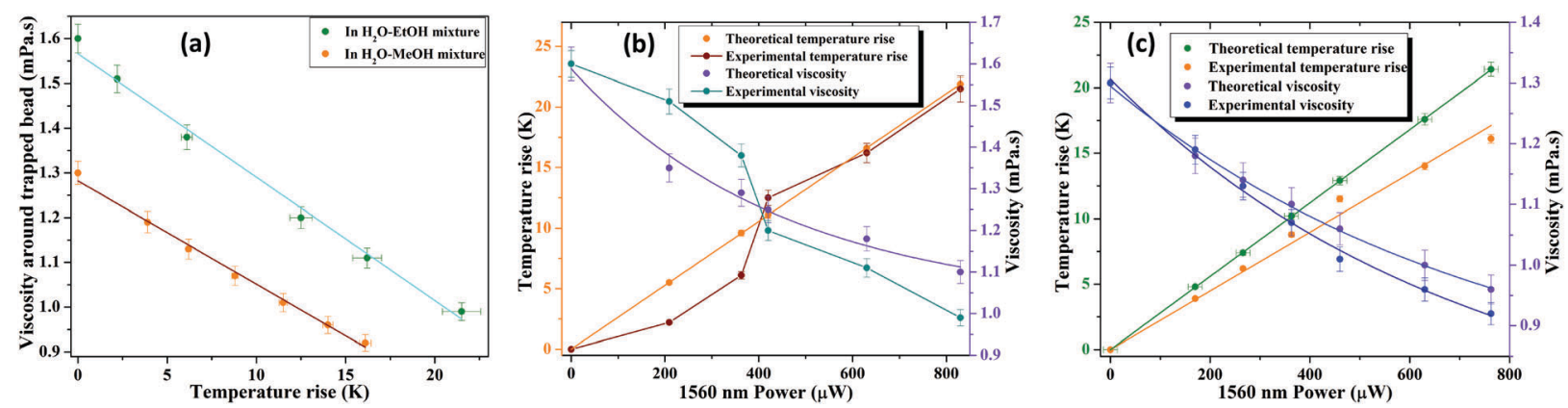

Fig. 3 (a) Experimental data for viscosity versus temperature rise (scatter plot) around the trapped bead and the corresponding linear fitting (solid line). The comparison of our theoretically corrected data for temperature rise (left side $Y$ axis) and viscosity (right side $Y$ axis) with experimental data in the respective (b) water-ethanol mixture and (c) water-methanol mixture. 
Here C1 represents pure water while the other parameters are the same as explained in the Theoretical section. In pure water, the theoretical gradient of temperature rise, calculated from eqn (2), is $(\mathrm{d} T / \mathrm{d} P)_{\text {Theo }}=0.034 \mathrm{~K}_{\mu} \mathrm{W}^{-1}$, while the measured experimental gradient of temperature rise is $(\mathrm{d} T / \mathrm{d} P)_{\operatorname{Exp}}=$

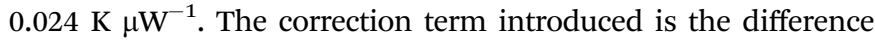
of these two values for binary solvent mixtures in eqn (8a). We plot the corrected theoretical temperature rise and the corresponding viscosity in Fig. $3 \mathrm{~b}$ and $\mathrm{c}$ for $\mathrm{H}_{2} \mathrm{O}-\mathrm{EtOH}$ and $\mathrm{H}_{2} \mathrm{O}-\mathrm{MeOH}$ respectively and compare them to the corresponding experimental data (also presented in Tables 2 and 3). For theoretical calculations, we first estimated the temperature rise $\left(T_{\max }-T_{0}(\mathrm{~K})\right)$ from applied $1560 \mathrm{~nm}$ pulse laser fluence using eqn (2), which is converted via eqn (5) to measure the surrounding viscosity. The corrected $\Delta T_{\text {theo }}$ is evaluated from eqn (8a), which is again transformed to measure the corrected viscosity using eqn (5).

It is important to note from Fig. $3 \mathrm{~b}$ that we observed a very unusual experimental behaviour of temperature rise $(\Delta T)$ and viscosity $(\eta)$ in the $\mathrm{H}_{2} \mathrm{O}$-EtOH binary mixture. This is in spite of the fact that the theoretical temperature rise $(\Delta T)$ followed a linear increase with $1560 \mathrm{~nm}$ laser power, and the viscosity $(\eta)$ followed the Vogel equation. This unusual behaviour is due to the loss of the individual properties of the constituent liquids in the binary mixtures, which is better explained in terms of non-ideal behaviour of the binary mixture as presented in Fig. 4. Fig. 4a shows the comparative plot of the slope of $1 / f_{\mathrm{C}}$ with $1560 \mathrm{~nm}$ laser power for water with methanol as well as ethanol. The distinct behaviour of the nature of the trap is
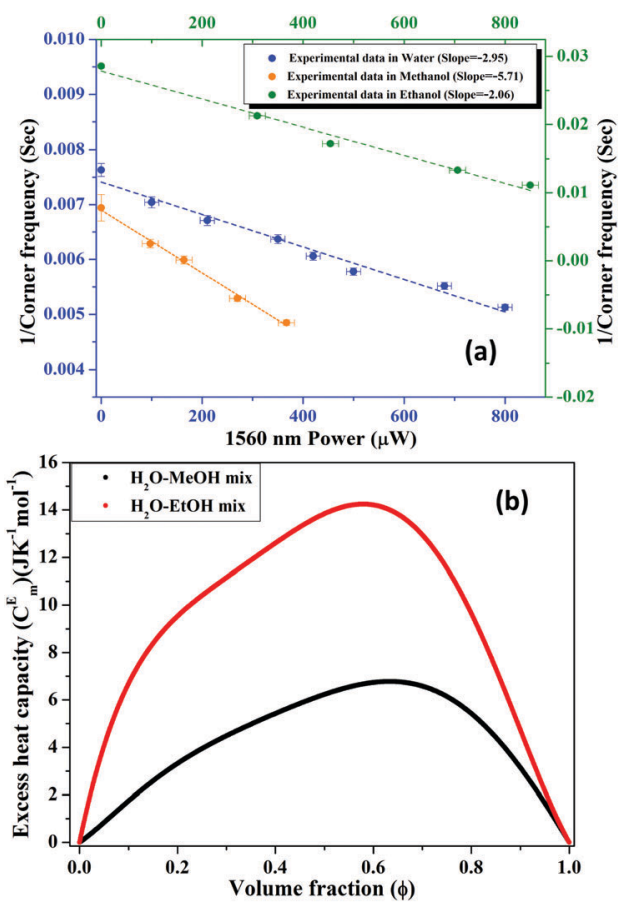

Fig. 4 (a) Comparative plot of inverse corner frequency versus power of a $1560 \mathrm{~nm}$ femtosecond laser with linear fitting (solid line) of water with pure $\mathrm{MeOH}$ and pure EtOH. (b) The comparison between excess heat capacity of $\mathrm{H}_{2} \mathrm{O}-\mathrm{MeOH}$ (black line) and $\mathrm{H}_{2} \mathrm{O}-\mathrm{EtOH}$ (red line) binary mixtures at $25^{\circ} \mathrm{C}$. evident from the distinct slopes in Fig. 4a for the three pure liquids, i.e., water (-2.95), methanol (-5.71) and ethanol (2.06), which also conform to the trend in the respective viscosities $(\eta)$ of these pure liquids $\left(\eta_{\mathrm{EtOH}}>\eta_{\mathrm{H}_{2} \mathrm{O}}>\eta_{\mathrm{MeOH}}\right)$. These numbers are indicative of the fact that the intermolecular forces in pure water and in pure ethanol are more similar as compared to the intermolecular forces in pure methanol. This would mean that in the case of binary mixtures of water with ethanol or methanol, the water-ethanol mixture would overall have more intermolecular force as compared to that of water-methanol. To further ratify our conjecture obtained from Fig. 4a, we plotted the comparative excess heat capacity in Fig. $4 \mathrm{~b}$, which shows that for a $10 \%$ volume mixture of $\mathrm{H}_{2} \mathrm{O}-\mathrm{MeOH}$ and $\mathrm{H}_{2} \mathrm{O}-\mathrm{EtOH}$, the excess heat capacity, $C_{\mathrm{p} \text {,mix }}^{\mathrm{E}}$, of the water-ethanol mixture (6.69 $\mathrm{J} \mathrm{K}^{-1} \mathrm{~mol}^{-1}$ ) is higher than that of the water-methanol mixture $\left(1.74 \mathrm{~J} \mathrm{~K}^{-1} \mathrm{~mol}^{-1}\right)$.

Since excess heat capacity is the measure of deviation from the ideal nature of mixtures, water-ethanol shows a higher deviation from ideal behaviour as compared to water-methanol. This corresponds to our observation from Fig. 3 that the watermethanol mixture follows the expected trend of $1560 \mathrm{~nm}$ laser heating while the water-ethanol mixture shows deviation.

The isobaric molar heat capacity of the binary mixture can be expressed by eqn (9) and (10). Thus, the excess heat capacity can be calculated by using eqn (11): ${ }^{67}$

$$
\left(\frac{\partial H_{\mathrm{m}, \mathrm{mix}}}{\partial T}\right)_{\mathrm{p}}=X_{\mathrm{C} 1}\left(\frac{\partial H_{\mathrm{m}, \mathrm{C} 1}}{\partial T}\right)_{\mathrm{p}}+X_{\mathrm{C} 2}\left(\frac{\partial H_{\mathrm{m}, \mathrm{C} 2}}{\partial T}\right)_{\mathrm{p}}+\left(\frac{\partial H_{\mathrm{m}}^{\mathrm{E}}}{\partial T}\right)_{\mathrm{p}}
$$

$$
\begin{gathered}
C_{\mathrm{p}, \mathrm{m}, \text { mix }}=X_{\mathrm{C} 1} C_{\mathrm{p}, \mathrm{C} 1}+X_{\mathrm{C} 2} C_{\mathrm{p}, \mathrm{C} 2}+C_{\mathrm{p}, \text { mix }}^{\mathrm{E}} \\
C_{\mathrm{p}, \text { mix }}^{\mathrm{E}}=\phi(1-\phi) \sum_{i=1}^{n} a(1-2 \phi)^{i-1}
\end{gathered}
$$

where $\mathrm{C} 2$ represents the second component of the binary mixture with water, $H$ is the enthalpy, $\varphi$ is the volume fraction of water and the " $a$ " value is used from ref. 67 .

Thus, our experimental results follow our theoretical framework that we have developed for the measurement of sub-micron volume temperatures as well as viscosity. For trapping polystyrene beads, individual solvents like $\mathrm{H}_{2} \mathrm{O}, \mathrm{MeOH}$ and EtOH are good solvents and they are also miscible among each other. ${ }^{68}$ We report our findings for a 90:10 volume mixture of $\mathrm{H}_{2} \mathrm{O}-\mathrm{EtOH}$ and $\mathrm{H}_{2} \mathrm{O}-\mathrm{MeOH}$. When the volume of alcohol in water-alcohol binary mixtures increases beyond $10 \%$, the viscosity of water-alcohol mixture increases rapidly until an equal volume mixture is reached. ${ }^{69}$ Higher viscosity results in lower corner frequency. Thus, the corner frequency shift due to the temperature rise will be small, which will decrease the experimental signal to noise ratio.

\section{Conclusions}

In this work, based on a non-invasive $780 \mathrm{~nm}$ femtosecond optical trap, we have demonstrated the control and measurement of direct in situ temperature at the solid-liquid interface, induced by a co-propagating $1560 \mathrm{~nm}$ pulsed laser. The viscosity 
as well as temperature around the trapped bead has been measured from the changes in corner frequency values. These measurements show that the thermal energy of solvents is transformed into the kinetic energy of the trapped bead. Correspondingly, we have developed a theoretical model that can explain these experimental results. Last but not the least, we were also able to probe the intermolecular interaction between two closely behaving solvent molecules.

\section{Acknowledgements}

We thank the Wellcome Trust Senior Research Fellowship (UK), the ISRO Science Technology Cell and DST, Govt. of India, for funding research presented here. DM thanks UGC, India, for the graduate fellowship. We thank D. Roy for useful discussions. We also thank Mrs S. Goswami for language editing of this article.

\section{References}

1 A. Ashkin, J. M. Dziedzic, J. E. Bjorkhom and S. Chu, Opt. Lett., 1986, 11, 288-290.

2 A. Ashkin, Optical Trapping and Manipulation of Neutral Particles Using Lasers: A Reprint Volume with Commentaries, World Scientific, 2006.

3 Q. Xing, F. Mao, L. Chai and Q. Wang, Opt. Laser Technol., 2004, 36, 635-639.

4 B. Agate, C. T. A. Brown, W. Sibbett and K. Dholakia, Opt. Express, 2004, 12, 3011-3017.

5 A. K. De, D. Roy, A. Dutta and D. Goswami, Appl. Opt., 2009, 48, G33-G37.

6 D. Roy, A. K. De and D. Goswami, Appl. Opt., 2015, 54, 7002-7006.

7 A. Schönle and S. W. Hell, Opt. Lett., 1998, 23, 325-327.

8 P. Kumar and D. Goswami, J. Phys. Chem. C, 2014, 118, 14852-14859.

9 D. Mondal and D. Goswami, Biomed. Opt. Express, 2015, 6, 3190-3196.

10 D. Mondal and D. Goswami, J. Nanophotonics, 2016, 10, 026013.

11 A. Ashkin and J. M. Dziedzic, Science, 1987, 235, 1517-1520.

12 S. M. Block, D. F. Blair and H. C. Berg, Nature, 1989, 338, 514-518.

13 A. Ashkin and J. M. Dziedzic, Proc. Natl. Acad. Sci. U. S. A., 1989, 86, 7914-7918.

14 A. Ashkin, J. M. Dziedzic and T. Yamane, Nature, 1987, 330, 769-771.

15 G. Herzberg, Infrared and Raman Spectra, D. Van Nostrand: Princeton, 1945.

16 C. L. Braun and S. N. Smirnov, J. Chem. Educ., 1993, 70, 612-614.

17 A. I. Bishop, T. A. Nieminen, N. R. Heckenberg and H. Rubinsztein-Dunlop, Phys. Rev. Lett., 2004, 92, 198104.

18 A. Pommella, V. Preziosi, S. Caserta, J. M. Cooper, S. Guido and M. Tassieri, Langmuir, 2013, 29, 9224-9230.

19 M. Tassieri, F. D. Giudice, E. J. Robertson, N. Jain, B. Fries, R. Wilson, A. Glidle, F. Greco, P. A. Netti, P. L. Maffettone, T. Bicanic and J. M. Cooper, Sci. Rep., 2015, 5, 8831.
20 Y. M. Rhee and V. S. Pande, J. Phys. Chem. B, 2008, 112, 6221-6227.

21 D. de Sancho, A. Sirur and R. B. Best, Nat. Commun., 2014, 5, 4307.

22 M. Guoa, Y. Xub and M. Gruebelea, Proc. Natl. Acad. Sci. U. S. A., 2012, 109, 17863-17867.

23 M. Karplus and J. A. Mccammon, Nat. Struct. Mol. Biol., 2002, 9, 646-652.

24 R. M. Daniel, M. E. Peterson, M. J. Danson, N. C. Price, S. M. Kelly, C. R. Monk, C. S. Weinberg, M. L. Oudshoorn and C. K. Lee, Biochem. J., 2010, 425, 353-360.

25 M. L. Begasse, M. Leaver, F. Vazquez, S. W. Grill and A. A. Hyman, Cell Rep., 2015, 10, 647-653.

26 G. E. Uhlenbeck and L. S. Ornstein, Phys. Rev., 1930, 36, 823-841.

27 M. C. Wang and G. E. Uhlenbeck, Rev. Mod. Phys., 1945, 17, 323-342.

28 A. Einstein, Ann. Phys., 1905, 322, 549-560.

29 F. Reif, Fundamentals of statistical and thermal physics, McGraw-Hill, Sydney, 1965.

30 T. G. Mason, K. Ganesan, J. H. van Zanten, D. Wirtz and S. C. Kuo, Phys. Rev. Lett., 1997, 97, 3282-3285.

31 F. C. MacKintosha and C. F. Schmidt, Curr. Opin. Colloid Interface Sci., 1999, 4, 300-307.

32 M. Atakhorrami, J. I. Sulkowska, K. M. Addas, G. H. Koenderink, J. X. Tang, A. J. Levine, F. C. MacKintosh and C. F. Schmidt, Phys. Rev. E: Stat., Nonlinear, Soft Matter Phys., 2006, 73, 061501.

33 A. Yao, M. Tassieri, M. Padgett and J. Cooper, Lab Chip, 2009, 9, 2568-2575.

34 T. Kalwarczyk, N. Zie-bacz, A. Bielejewska, E. Zaboklicka, K. Koynov, J. Szymanski, A. Wilk, A. Patkowski, J. Gapinski, H.-J. Butt and R. Hozyst, Nano Lett., 2011, 11, 2157-2163.

35 G. Charras and E. Sahai, Nat. Rev. Mol. Cell Biol., 2014, 15, 813-824.

36 Y. Pang and R. Gordon, Nano Lett., 2012, 12, 402-406.

37 D. Mondal and D. Goswami, SPIE Proc., 2015, 9548, 95481N.

38 P. Kumar, A. Khan and D. Goswami, Chem. Phys., 2014, 441, 5-10.

39 J. H. Lienhard IV and J. H. Lienhard V, A Heat Transfer Textbook, Phlogiston Press, Cambridge, Massachusetts, USA, 3rd edn, 2004.

40 Y. Liu, G. J. Sonek, M. W. Berns and B. J. Tromberg, Biophys. J., 1996, 71, 2158-2167.

41 H. S. M. Coxeter and S. L. Greitzer, Geometry Revisited, The Mathematical Association of America, Washington, DC, 1967.

42 http://www.olympus-ims.com/en/microscope/terms/luminous_ flux/(accessed 14 July, 2016).

43 X. E. Lin, in Proceedings of the 1999 Particle Accelerator Conference, (IEEE, 1999), 1429-1431.

44 T. A. Scott, J. Phys. Chem., 1946, 50, 406-412.

45 J. Ortega, J. Chem. Eng. Data, 1982, 27, 312-317.

46 J.-P. E. Grolier, Fluid Phase Equilibrium, 1981, 6, 283-287.

47 G. C. Benson, P. J. D'Arcy and O. K. Kiyohara, J. Solution Chem., 1980, 9, 931-938. 
48 J. D. Raal and R. L. Rijsdijk, J. Chem. Eng. Data, 1981, 26, 351-359.

49 T. Al-Shemmeri, Engineering Fluid Mechnanics, Ventus Publishing ApS, 2012.

50 Dartmund Data Bank (DDB), http://ddbonline.ddbst.de/ VogelCalculation/VogelCalculationCGI.exe.

51 J. Kendall and K. P. Monroe, J. Am. Chem. Soc., 1917, 39, 1787-1802.

52 M. Schnell and B. A. Wolf, J. Rheol., 2000, 44, 617-628.

53 R. Mertsch and B. A. Wolf, Ber. Bunsenges. Phys. Chem., 1994, 98, 1275-1280.

54 S. N. Damask, Polarization Optics in Telecommunications, Springer-Verlag, New York, 2005.

55 R. Radünz, D. Rings, K. Kroy and F. Cichos, J. Phys. Chem. A, 2009, 115, 1674-1677.

56 D. Rings, R. Schachoff, M. Selmke, F. Cichos and K. Kroy, Phys. Rev. Lett., 2010, 105, 090604.

57 L. Joly, S. Merabia and J.-L. Barrat, Europhys. Lett., 2011, 94, 50007.

58 A. C. Richardson, S. N. S. Reihani and L. B. Oddershede, Opt. Express, 2008, 16, 15709-15717.

59 R. Kubo, M. Toda and N. Hashitsume, Statistical Physics, Springer, Heidelberg, 1985, vol. 2.
60 W. H. Press, B. P. Flannery, S. A. Teukolsky and W. T. Vetterling, Numerical Recipes. The Art of Scientific Computing, Cambridge University Press, Cambridge, ch. 12.0, 1998.

61 K. Berg-Sørensen and H. Flyvbjerg, Rev. Sci. Instrum., 2004, 75, 594-612.

62 G. Pesce, A. Sasso and S. Fusco, Rev. Sci. Instrum., 2005, 76, 115105.

63 S. F. Tolić-Nørrelykkea, E. Schäffer, J. Howard, F. S. Pavone, F. Jülicher and H. Flyvbjerg, Rev. Sci. Instrum., 2006, 77, 103101.

64 I.-M. Tolić-Nørrelykkea, K. Berg-Sørensen and H. Flyvbjerg, Comput. Phys. Commun., 2004, 159, 225-240.

65 H. Mao, J. R. Arias-Gonzalez, S. B. Smith, I. Tinoco, Jr. and C. Bustamante, Biophys. J., 2005, 89, 1308-1316.

66 A. Marcano, C. Loper and N. Melikechi, J. Opt. Soc. Am. B, 2002, 19, 119-124.

67 G. C. Benson, P. J. D'Arcy and O. Kiyohara, J. Solution Chem., 1980, 9, 931-938.

68 D. Mukherji, C. M. Marques and K. Kremer, Nat. Commun., 2014, 5, 4882.

69 E. J. W. Wensink, A. C. Hoffmann, P. J. van Maaren and D. van der Spoel, J. Chem. Phys., 2003, 119, 7308-7317. 